\title{
Machine Learning to Find Areas of Rotors Sustaining Atrial Fibrillation From the ECG
}

\author{
Giorgio Luongo ${ }^{1}$, Luca Azzolin ${ }^{1}$, Massimo W Rivolta ${ }^{2}$, Tiago P Almeida ${ }^{3}$, Juan Pablo Martínez ${ }^{4}$, \\ Diogo C Soriano ${ }^{5}$, Olaf Dössel ${ }^{1}$, Roberto Sassi ${ }^{2}$, Pablo Laguna ${ }^{4}$, Axel Loewe ${ }^{1}$ \\ ${ }^{1}$ Institute of Biomedical Engineering, Karlsruhe Institute of Technology (KIT), Karlsruhe, Germany \\ ${ }^{2}$ Dipartimento di Informatica, Università degli Studi di Milano, Milan, Italy \\ ${ }^{3}$ Department of Cardiovascular Sciences, University of Leicester, Leicester, UK \\ ${ }^{4}$ I3A, Universidad de Zaragoza, and CIBER-BNN, Zaragoza, Spain \\ ${ }^{5}$ Engineering, Modelling and Applied Social Sciences Centre, ABC Federal University, São Bernardo \\ do Campo, Brazil
}

\begin{abstract}
Atrial fibrillation $(A F)$ is the most frequent irregular heart rhythm due to disorganized atrial electrical activity, often sustained by rotational drivers called rotors. The non-invasive localization of $A F$ drivers can lead to improved personalized ablation strategy, suggesting pulmonary vein $(P V)$ isolation or more complex extra$P V$ ablation procedures in case the driver is on other atrial regions. We used a Machine Learning approach to characterize and discriminate simulated single stable rotors (1R) location: PVs, left atrium (LA) excluding the $P V s$, and right atrium (RA), utilizing solely non-invasive signals (i.e., the 12-lead ECG). IR episodes sustaining $A F$ were simulated. 128 features were extracted from the signals. Greedy forward algorithm was implemented to select the best feature set which was fed to a decision tree classifier with hold-out cross-validation technique. All tested features showed significant discriminatory power, especially those based on recurrence quantification analysis (up to $80.9 \%$ accuracy with single feature classification). The decision tree classifier achieved $89.4 \%$ test accuracy with 18 features on simulated data, with sensitivities of $93.0 \%, 82.4 \%$, and $83.3 \%$ for $R A, L A$, and $P V$ classes, respectively. Our results show that a machine learning approach can potentially identify the location of $1 R$ sustaining AF using the 12-lead ECG.
\end{abstract}

\section{Introduction}

Atrial fibrillation (AF) is the most common sustained arrhythmia in clinical practice and a leading cause of hospitalization and death [1]. This arrhythmia is often sustained by localized functional reentrant circuits called rotors, characterized by curved wavefronts and wavetails that meet each other at a singularity point [2]. One common therapy to terminate AF is ablation. Typically, "triggers" that start AF and/or the "substrate" that participates in its perpetuation are targeted during ablation. However, it remains unclear which of the two approaches is the most effective for treating AF, specially in advanced stages of the disease. Narayan et al. showed that it is important to localize and ablate rotors, focal sources drivers or organizing sources of fibrillation to terminate AF [3]. Additionally, triggers and sustaining mechanisms are often localized in the pulmonary veins (PVs) [4]. Thus, $\mathrm{PV}$ isolation (PVI) is the first ablation procedures applied to try to terminate $\mathrm{AF}$.

In this preliminary work, we sought to characterize and identify single stable rotors (1R) located near the PVs, on extra-PV left atrium (LA) areas, and on right atrium (RA) areas by using 12-lead electrocardiogram (ECG) in a simulation study. This non-invasive method could help guide ablation procedures, highlighting atrial regions that may be important in the AF perpetuation, and hence targets for ablation. In case of rotors identified within the PVs, the application of a priori invasive and time-consuming electrophysiologic mapping procedures could be avoided, proceeding directly with PVI.

\section{Methods}

\subsection{Simulations}

$1 \mathrm{R}$ episodes sustaining AF were simulated using the phase singularity distribution method on a volumetric atrial model built from clinical data, as reported in [5]. Briefly, the phase singularities were placed in 300 uniformly distributed points in the atria, and $3 \mathrm{~s}$ of activation were 
computed. Only the cases with $1 \mathrm{R}$ episodes that kept going for the whole simulation time were considered for further analysis. This led to unbalanced data generation. As result of the monodomain simulation, the transmembrane voltage was used to calculate the body surface potential map (BSPM) on 8 different torso models generated from segmented MRI data of healthy male and female subjects (Fig. 1), [5]. From the BSPM, the 12-lead ECG was extracted with a length of $3 \mathrm{~s}$ (Fig. 1). Only f-waves without the QRS-T complex composed the 12-lead ECG, since the ventricles were not included in the simulations. 440 sets of 12-lead ECG formed the final dataset (40 ECGs with 1R located in the PVs, 112 in extra-PV LA areas, and 288 in the RA).

\subsection{Feature extraction}

128 features were extracted from the the signals using several biosignal processing methods, such as: Hjort descriptors to analyse the spectral moments from the time signals [5]; recurrence quantification analysis (RQA) on vectocardiogram (VCG) [6], individual component RQA (icRQA), and spatial reduced RQA (srRQA) [7] to analyse the topological structure of multidimensional dynamical systems; ratio of the principal component analysis (PCA) eigenvalues, organization index, and spectral entropy to study the variability and stability of these mechanisms over time and frequency [5], [8], [9].

\subsection{Feature selection}

The feature set was selected with a greedy forward selection technique. Starting with an empty feature set, this algorithm added the feature which lead to the highest accuracy increase of the set at each iteration. The performances were based on the validation set. When the performance did not increase further, the algorithm was stopped. Candidate features with a correlation coefficient $>0.6$ with any of the features already included in the set have been removed to avoid possible correlation between features and redundancy of information into the set.

\subsection{Classification}

Due to its simplicity, a decision tree classifier was implemented for a 3 classes discrimination: PV rotors, extra-PV LA rotors, and RA rotors.

All extracted features were individually evaluated with a decision tree classifier and a leave-one-out cross-validation technique. Subsequently, with the feature set selected by the greedy technique, a multi-feature classification with hold-out cross-validation was performed $(70 \%, 15 \%$, and
Table 1. Three single features with the highest accuracy for PV vs. extra-PV LA vs. RA classification

\begin{tabular}{lc}
\hline \hline Feature & Accuracy (\%) \\
\hline$R_{V C G}^{R}$ & 80.9 \\
$E_{s r R Q A}^{D L}$ & 80.4 \\
$E_{i c R Q A_{4}}^{V L}$ & 80.0 \\
\hline \hline
\end{tabular}

$15 \%$ of the total dataset was randomly divided into training set, validation set, and test set, respectively). Sensitivity and specificity were calculated for each class considering the class at hand as positive and the remaining two classes as negative.

\subsection{Statistical analysis}

The ability of the features in separating the different classes was assessed using the the Kruskal-Wallis nonparametric one-way analysis of variance for a multi-class evaluation. $p$-values of less than 0.01 were considered statistically significant.

\section{Results}

\subsection{Features evaluation}

All features showed an individual and significant discriminatory power. Among them all, RQA's parameters have stood out particularly well. Indeed, the most discriminating 3 individual features were: the recurrence rate extracted from VCG $\left(R_{V C G}^{R}\right)$; the diagonal entropy extracted with srRQA $\left(E_{s r R Q A_{d}}^{D L}\right.$; and the vertical entropy extracted with icRQA $\left(E_{i c R Q A_{4}}^{V L}\right)$. Table 1 shows the accuracy singularly reached. These 3 features showed significantly higher values for 1R located in the PV class, followed by the extra-PV LA class, and the RA class, respectively (Fig. 2).

\subsection{Rotors location classification}

The decision tree classifier achieved $89.4 \%$ test accuracy with a feature set of 18 features, with sensitivity of $93.0 \%, 82.4 \%$, and $83.3 \%$, and a specificity of $95.2 \%$, $77.8 \%$, and $83.3 \%$ for RA, extra-PV LA, and PV class respectively. 5 selected features have been calculated using RQA methods (including the 3 best features showed in section 3.1). 11 selected features have been extracted from the ratio of the PCA eigenvalues approach. Finally, 2 selected features belonged to the Hjort descriptors. Table 2 shows the test-set confusion matrix obtained from the decision tree using the feature set (class LA represents class extra-PV LA). 
A.1

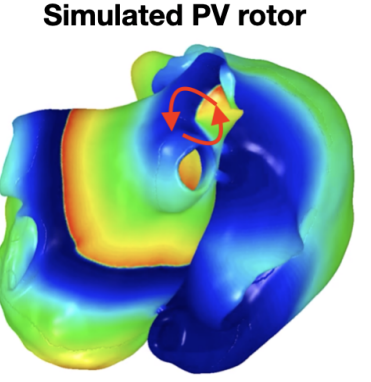

A.2 Body surface potential map

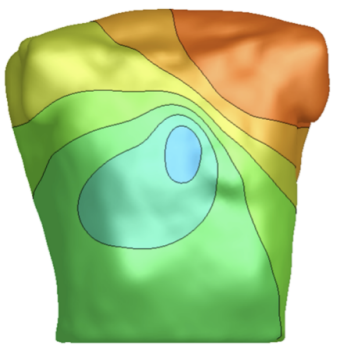

A.3

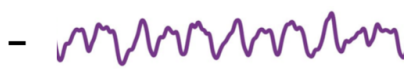

- MAMMA

$\Sigma$

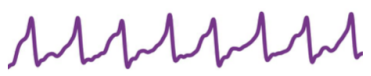

B.1 Simulated extra-PV LA rotor

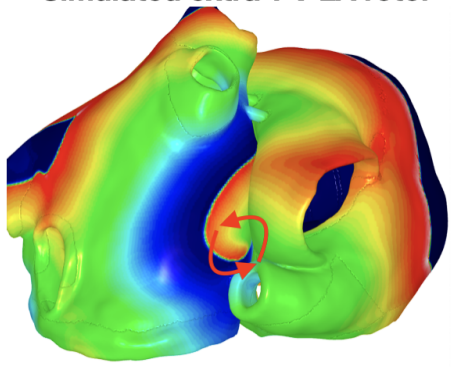

B.2 Body surface potential map

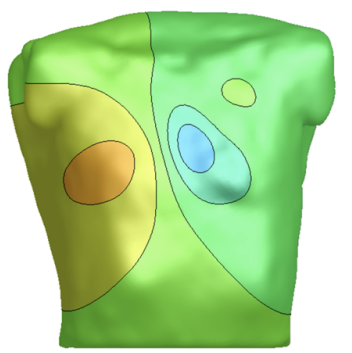

B.3

Electrocardiogram

- Marrorrerrar

= MMroburw

- Mrromarr

\section{C.1}

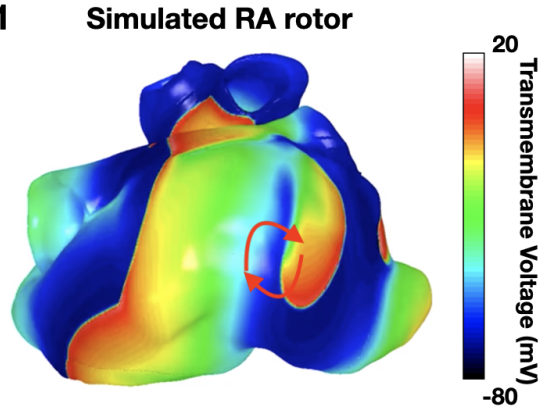

C.2 Body surface potential map

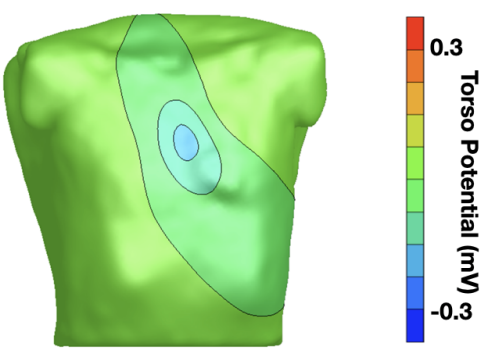

\section{C.3 Electrocardiogram}

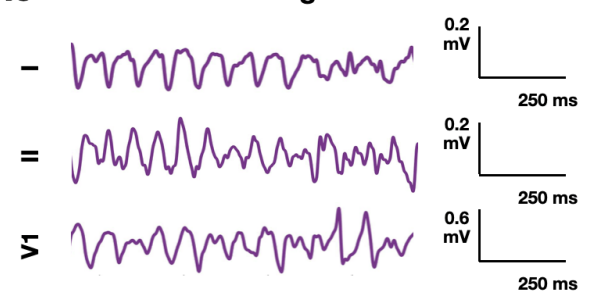

Figure 1. A.1: Simulated PV rotor. B.1: Simulated extra-PV LA rotor. C.1 : Simulated RA rotor. The red arrows show the rotor position and direction. A.2-B.2-C.2: BSPMs of one of the 8 torso models generated from MRI. The torso potential was obtained by solving the forward problem of electrophysiology from the simulated TMV on the atria. A.3-B.3-C.3: Example of the f-wave for lead I, II, and V1 from the 12-lead ECG signals extracted from the BSPMs.

Table 2. Test-set confusion matrix for RA, extra-PV LA, and PV rotors classification

\begin{tabular}{ll|ccc} 
& & \multicolumn{3}{c}{ True class } \\
& & RA & LA & PV \\
\cline { 2 - 5 } Predicted class & RA & 40 & 2 & 0 \\
& LA & 3 & 14 & 1 \\
& PV & 0 & 1 & 5
\end{tabular}

\section{Discussion and Conclusions}

Simulations provide ideal and controlled scenarios where the ground truth for AF perpetuation sustained by $1 \mathrm{R}$ is known in all the cases. This allows the analysis of each simulation without the influence of secondary, or unknown, mechanisms, e.g., other simultaneous rotors.
The RQA's parameters showed to be key features for this classification (Table 1). Probably due to their sensitivity in detecting changes in the dynamic behavior of these mechanisms. In fact, looking also at the example ECGs in Fig. 1A-B-C.3, our simulations have shown that the ECG signals are more irregular in cases when $1 \mathrm{R}$ is not in the PVs area. This information was also quantified by some RQA parameters, having significantly higher values for the RA class, followed by the extra-PV LA class, ending with lower values for the PV class (Fig. 2). This can be seen as confirmation of what was suggested in a previous study [5].

As mentioned above, the ECG signals in the case of $1 \mathrm{R}$ not located in the PVs areas are more irregular. All features extracted were aimed at detecting these irregularities and differences between classes. The results obtained with the hold-out cross-validation showed that an automatic 


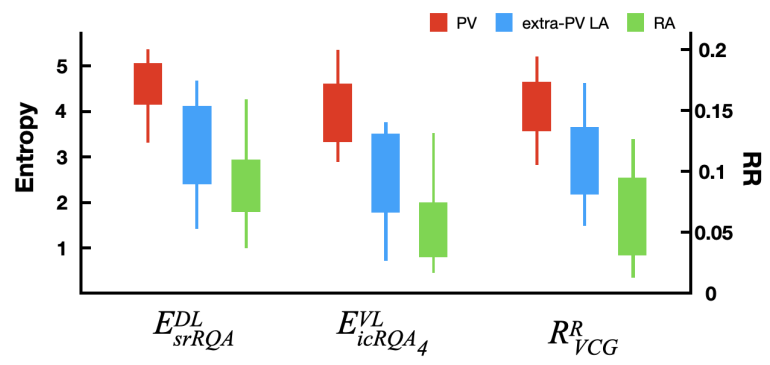

Figure 2. Boxplots of the 3 single features with the highest accuracy for PV (red) vs. extra-PV LA (blue) vs. RA (green) rotor location classification. All features are statistically different between the classes with $\mathrm{p}<0.01$

classifier with the features extracted in this work can potentially identify the area where a $1 \mathrm{R}$ is located using the 12-lead ECG.

The high sensitivity and specificity values obtained for all classes show that this automatic classification method categorizes most of the cases in analysis into the correct class. Therefore, if $1 \mathrm{R}$ was classified as a PV case, doctors could proceed directly with a PVI by cryoablation, without using a priori mapping system. In the other cases, a radio frequency ablation procedure with a previous mapping of the electrical activity of the atrium of interest would be required.

The use of a non-invasive technique (i.e., 12-lead ECG), in combination with machine learning approaches, may directly suggest to the doctor the atrial regions that may be important in the AF perpetuation, and hence targets for ablation. Further tests on clinical data, labelled by inspecting the local activation maps, are necessary to effectively assess the proposed approach. A subsequent study to predict the outcome of PVI in cases where the AF driver is in PV is ongoing.

In conclusion, this work could be extended with a prior characterization of different AF driver mechanisms and AF complexity analysis. Several and more robust classification algorithms can be tested and more simulations can be generated with different atrial models.

\section{Acknowledgments}

The authors thank Deborah Nairn for her valuable suggestions. Research supported by the European Union's Horizon 2020 research and innovation programme under the Marie Sklodowska-Curie grant agreement No.766082 (MY-ATRIA). TPA received support from the British Heart Foundation (PG/18/33/33780 and BHF Research Accelerator). All authors confirm that they have no other relationships relevant to the contents of this paper to disclose.

\section{References}

[1] Calkins H, et al. $2012 \mathrm{hrs} / \mathrm{ehra} / \mathrm{ecas}$ expert consensus statement on catheter and surgical ablation of atrial fibrillation: recommendations for patient selection, procedural techniques, patient management and followup, definitions, endpoints, and research trial design. Heart Rhythm 2012;9(4):632-696.

[2] Pandit S, Jalife J. Rotors and the dynamics of cardiac fibrillation. Circ Res 2013;112:849-862.

[3] Narayan SM, Baykaner T, Clopton P, Schricker A, Lalani GG, Krummen DE, Shivkumar K, Miller JM. Ablation of rotor and focal sources reduces late recurrence of atrial fibrillation compared to trigger ablation alone. J Am Coll Cardiol 2014;63(17):1761-1768.

[4] Haissaguerre M, Jais P, Shah DC, Takahashi A, Hocini M, Quiniou G, Garrigue S, Mouroux AL, Metayer PL, Clémenty J. Spontaneous initiation of atrial fibrillation by ectopic beats originating in the pulmonary veins. N Engl J Med 1998; 339:659-666.

[5] Luongo G, Azzolin L, Rivolta MW, Sassi R, Laguna P, Dössel O, Loewe A. Non-invasive identification of atrial fibrillation driver location using the 12-lead ECG: Pulmonary vein rotors vs. other locations. IEEE 2020 EMB Conference 2020;410-413.

[6] Yang H. Multiscale recurrence quantification analysis of spatial cardiac vectocardiogram signals. IEEE Trans Biomed Eng 2011;58(2):339-347.

[7] Luongo G, Schuler S, Luik A, Almeida TP, Soriano DC, Dössel O, Loewe A. Non-invasive characterization of atrial flutter mechanisms using recurrence quantification analysis on the ECG: a computational study. IEEE Trans Biomed Eng 2020; ;

[8] Jarman JWE, Wong T, Kojodjojo P, Spohr H, Davies JER, Roughton M, Francis DP, Kanagaratnam P, Dphil M, Markides V, Davies DW, Peters NS. Organizational index mapping to identify focal sources during persisent atrial fibrillation. J Cardiovasc Electrophysiol 2014;25(4):355363.

[9] Vakkuri A, Yli-Hankala A, Talja P, Mustola S, TolvanenLaasko H, Sampson T, Viertiö-Oja H. Time-freuqency balanced spectral entropy as a measure of anesthetic drug effect in central nervous system during sevoflurane, propofol, and thiopental anesthesia. Acta Anaesthesiol Scand 2004; 48(2):145-153.

Address for correspondence:

Giorgio Luongo, Karlsruhe Institute of Technology (KIT)

Fritz-Haber-Weg 1, 76131 Karlsruhe, Germany

publications@ibt.kit.edu 